\title{
Use of Extraoral Multidirectional Distractor as an External Pin Fixator: A Novel Technique in the Management of Comminuted Mandibular Fracture
}

\author{
Ayman F. Hegaba, , Mohammed Abd El-Akher ${ }^{\mathrm{a}}$
}

\begin{abstract}
Backgroud: The treatment of comminuted fractures of the mandible is one of the most complex situations that the oral and maxillofacial surgeon is facing within his practice. The aim of the current study was to evaluate the use of extraoral multidirectional distractor as an external pin fixator in comminuted mandibular fracture.
\end{abstract}

Methods: Five male patients (age range from 23 to 37 years) with comminuted mandibular fractures were included in this study. Under general anesthesia, the extraoral multidirectional distractor was applied and used to reduce the fracture segments without intermaxillary fixation followed by checking the occlusion for any disturbance that can be corrected by the distractor. After evaluation of the reduction of the fracture segments by panoramic and lateral mandibular X-ray views, self-cure acrylic resin was used to stabilize the pins and the distractor was removed in the outpatient clinic. The stabilization period ranges from 8 to 10 weeks.

Results: The use of extraoral multidirectional distractor as an external pin fixator was successful in establishing good reduction of the fracture line with stable occlusion without open bites or malocclusion in four patients. One patient showed mild discrepancies of the occlusion that was corrected by spot grinding of the molar teeth in the outpatient clinic. The patients showed good reduction of the comminuted fracture and good functional results without infection. No nerve or root tooth injury was observed. No patients required revision of fixation for malreduction, non-union or malocclusion.

Conclusions: The application of extraoral multidirectional distractor as an external pin fixator can be considered as a potentially effective, easy and reliable technique for the reduction of the comminuted mandibular fracture. Further studies to evaluate the efficacy of the extraoral multidirectional distractor as an external pin fixator

Manuscript accepted for publication July 28, 2014

${ }^{a}$ Department of Oral and Maxillofacial Surgery, Faculty of Dental Medicine, Al-Azhar University, Cairo, Egypt

${ }^{\mathrm{b}}$ Corresponding Author: Ayman F. Hegab, Department of Oral and Maxillofacial Surgery, Faculty of Dental Medicine, Al-Azhar University, Cairo, Egypt. Email: hegab@mail.com

doi: http://dx.doi.org/10.14740/jcs235w are recommended on a large number of patients.

Keywords: Comminuted mandibular fracture; Extraoral distractor; External pin fixation

\section{Introduction}

Comminuted fractures of the mandible generally affect young, adult men, and have a widely varying incidence and etiology according to the socio-economic conditions of the location. In countries where there is armed conflict, the incidence of this type of fracture is high, and its main etiologic agent is injuries due to low-velocity gunshot injury. Among the fractures of the face, the mean incidence of this type of situation is low, around 2-6\%, and their main etiologic agent is motor car accidents. They are also caused by falls, interpersonal aggressions and sports. The body of the mandible is generally the most affected part, followed by the symphysis and the angle. Usually, there is only one area of comminution, but there may be other associated fractures in the mandible [1-5].

The treatment of comminuted fractures of the mandible is one of the most complex situations that the oral and maxillofacial surgeon is facing within his practice. Its treatment is a challenge, due to both the severity of the associated injuries that generally accompany this fracture, and the lack of consensus in the literature about what would be the most adequate method of treating it.

There are basically two distinct approaches for the treatment of comminuted fractures of the mandible. Closed reduction of the fracture (maxillomandibular fixation (MMF), external pin fixation, lingual splint with circumferential skeletal fixation) is the oldest and classical treatment for this injury. Those that defend it point out its advantage as being less morbidity, which would avoid stripping the periosteum of the fractured fragments, and thus the chances of necrosis and infection by tissue ischemia, since a large part of the bone tissue blood supply comes from the periosteum. Nevertheless, with the advent of modern anesthesia and antibiotics, the classical paradigm of closed treatment for comminuted fractures of the mandible began to be questioned since 1958, 

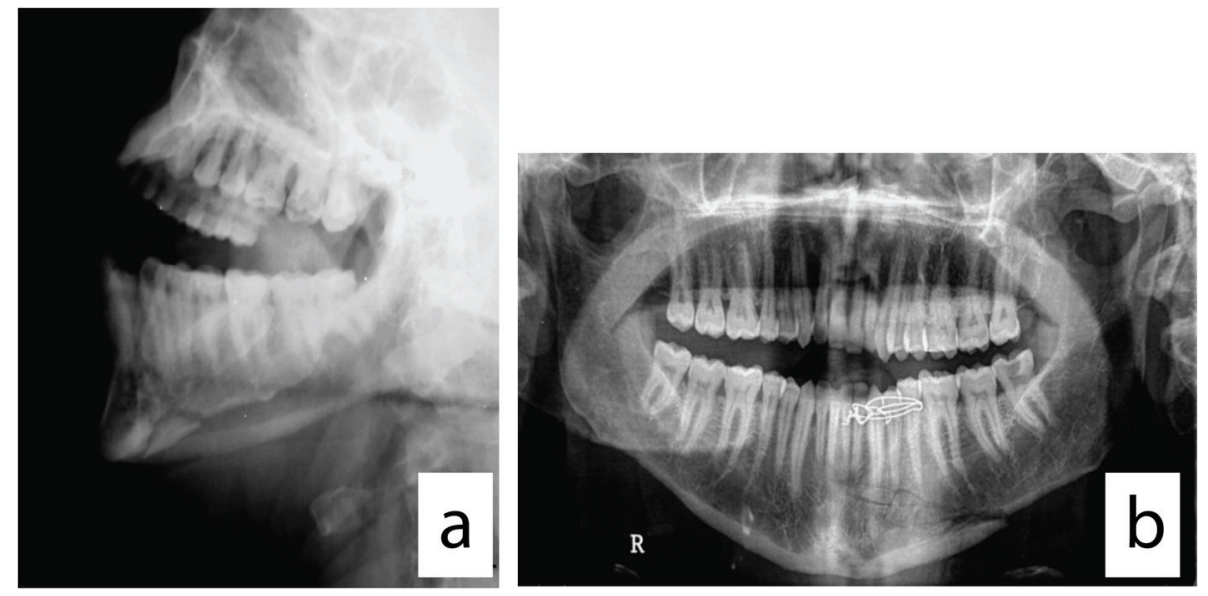

Figure 1. (a) Mandibular lateral oblique X-ray view for evaluation of the comminuted fracture. (b) Mandibular panoramic X-ray view for evaluation of the comminuted fracture.

when around 50 surgeons formed the Arbeitsgemeinschaft fur Osteosynthesefragen (AO) in Switzerland, which was later known in the United States as Association for Study of Internal Fixation (ASIF), and developed the technical and instrumental principles for open reduction and rigid internal fixation (ORIF). In accordance with the principles of the AO/ASIF, the goal of ORIF in the treatment of comminuted fractures of the mandible is to achieve undisturbed biological environment and restore the shape and early return to function without the adjunct use of MMF, by means of absolute immobilization of the bone fragments and primary bone repair, obtained with plates and bicortical screws [5, 6-9].

The external splinting of the mandible with pin fixation or with an external fixator appliance, respectively, is currently an unusual modality in fracture treatment with an indication confined to few problematic cases beyond usual routine. In the years between 1940 and 1950, external pin fixator was modified for its particular use in mandibular fractures and further on was propagated during two time spans (from 1940 to approximately 1975 and from 1985 to approximately
1995). However, with increasing popularity of rigid internal fixation methods in craniofacial trauma and reconstructive surgery, pin fixation began to disappear from the common repertoire and was even attributed to have a touch of relic [10].

\section{Materials and Methods}

After obtaining institutional approval (Al-Azhar University Hospital, Cairo, Egypt) and informed written consent, a case series study was conducted from May 2007 to June 2009. A careful clinical examination was performed externally and intraorally in sequential manner to include all regions of the face. All the patients were explained to the different treatment modalities: closed reduction, external pin fixation and ORIF using plates and screws. Five male patients from total 13 patients with comminuted mandibular fracture (bodyparasymphsial) opted external pin fixation. The other patients (five male and three female) did not accept this type of fixa-
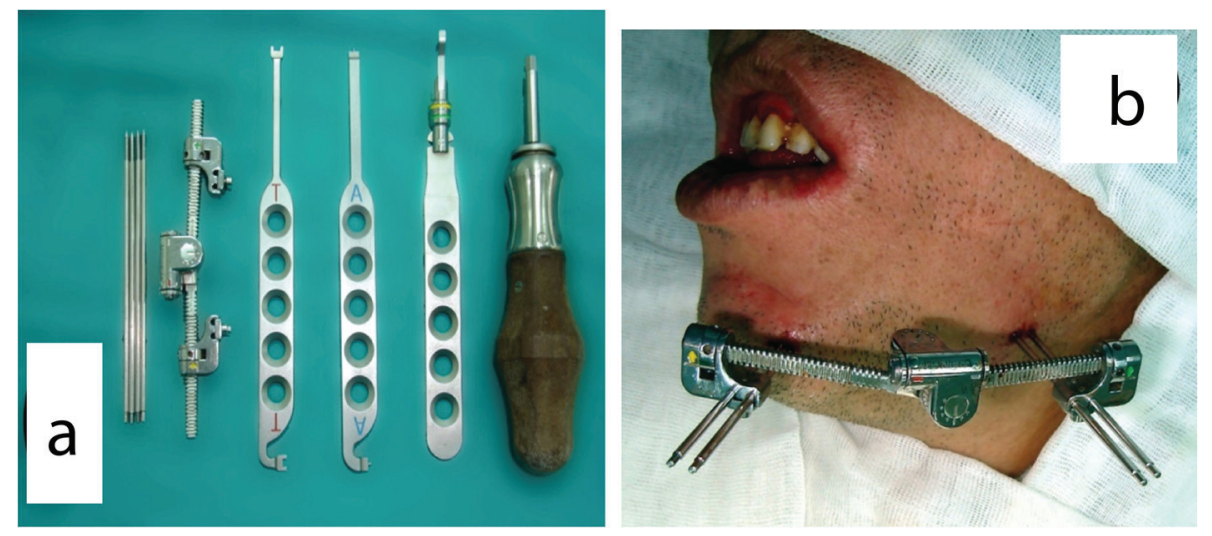

Figure 2. (a) The extraoral multidirectional distractor kit. (b) The distractor in place after reduction of the fracture. 


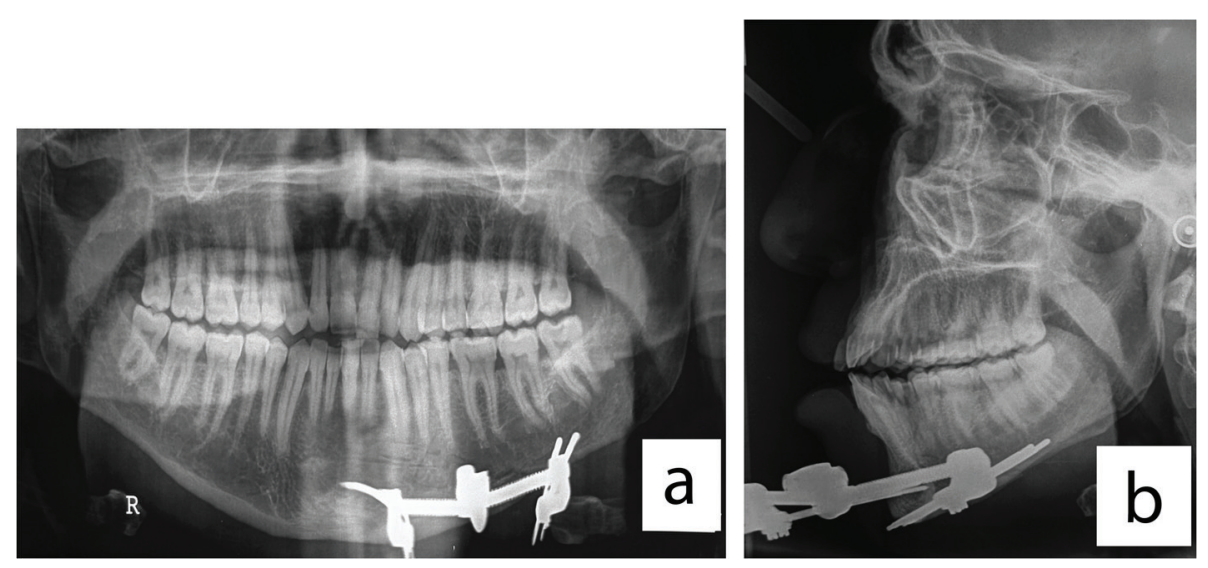

Figure 3. (a) Postoperative panoramic X-ray view showed good reduction and occlusion. (b) Postoperative panoramic and mandibular lateral X-ray views showed good reduction and occlusion.

tion for cosmetic causes and interference with the lifestyle of the patients and they have been excluded from this study. On admission, antibiotics are prescribed to prevent infection that could result in initial loss of bone and the operation was done within 3 - 4 days from the trauma. Radiographic evaluation of the fracture was done using panoramic X-ray or mandibular lateral views (Fig. 1a, b). The clinical findings were then correlated with diagnostic radiographic imaging.

Under general anesthesia, manual reduction of the fracture with occlusion guidance was used during the application of the distractor and insertion of the pins without intermaxillary fixation. Two pins in each side of the fracture line as close as possible but not less than $1 \mathrm{~cm}$ were inserted. The pin insertion was done through the trocar as a guide overlaying the safe zones to avoid injury of the vital structures. The self-drilling pins were loaded into the handle and driven into the bone, the handle was disengaged from the pin and the trocar was removed. The length of the threaded portion of the pins was chosen to attain bicortical engagement. The extraoral multidirectional distractor (Fig. 2a, b) was applied and used to reduce the fracture segments without intermaxillary fixation followed by checking the occlusion for any disturbances that can be corrected by the distractor. The operating time ranged from 60 to $90 \mathrm{~min}$. After evaluation of the reduction of the fracture segments by panoramic and lateral mandibular X-ray views (Fig. 3a, b), self-cure acrylic resin was used to stabilize the pins and the distractor was removed in the outpatient clinic. The stabilization period range from 8 to 10 weeks.

Daily care of the pin sites including regular cleansing with $70 \%$ alcohol and $0.2 \%$ chlorhexidine solution was carried out.

Functional results were evaluated according to the following criteria: 1) according to occlusion: good (neutrocclusion), fair (malocclusion not functionally significant) and poor (significant malocclusion); 2) according to reduction of fracture: good (no step deformity and good occlusion), fair (no step deformity and mild malocclusion) and poor (step deformity with malocclusion); 3) nerve or tooth root injury; 4) bony union: malunion, non-union (requiring revision of fixation) and good union; 5) infection; 6) trismus.

\section{Results}

All the patients healed well with this type of fixation. None of the patients had non-union.

Unlike intermaxillary fixation, there is no compromise
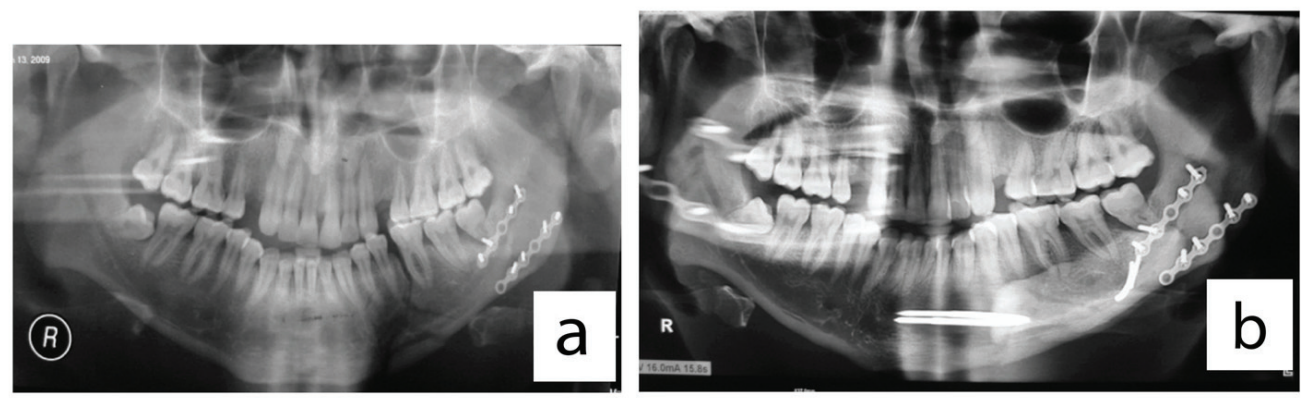

Figure 4. (a) Preoperative panoramic X-ray view of comminuted mandibular fracture and mini-plate fixation of old mandibular angle fracture. (b) Reduction of the fracture with mild occlusion discrepancies. 
to the airway and no special precautions for the release of the fixation. As the mouth can be opened during fracture healing, oral hygiene and patient nutrition were improved.

The use of extraoral multidirectional distractor as external pin fixator was successful in establishing a stable good occlusion (neutrocclusion) without open bites or malocclusion in four patients.

One patient showed mild discrepancies of the occlusion (fair occlusion) that was corrected by spot grinding of the molar teeth in the outpatient clinic (Fig. 4a, b). One patient showed mild trismus that improved within few days by muscle relaxant. The other cases showed good reduction of the comminuted fracture as showed by the X-ray and clinically by good occlusion and absence of step deformity at the inferior border of the mandible with good functional results. No infection at the pin sites or loosening of the pin was observed. No nerve or root tooth injury was observed. No patient required revision of fixation for malreduction, non-union or malocclusion. The total time of surgery ranged from 60 to 90 min eliminating the time needed for the intermaxillary fixation and the risk of puncture injury from wires.

\section{Discussion}

Patients with comminuted fractures of the mandible could be successfully treated by a number of methods. Closed treatment with MMF, external pin fixation, or open treatment using stable internal fixation devices, all worked when applied under the appropriate circumstance [6].

Theoretically, the mandibular external fixator could be used to achieve definitive bone healing. Nevertheless, the external fixator does not offer the same degree of the stability compared to the internal devices.

The use of extraoral multidirectional distractor as external pin fixator could be a potentially effective, reliable and simple method for mandibular reduction and provides a distinctive characteristic compared to the traditional external pin fixation. The extraoral multidirectional distractor allowed the dynamic evaluation of the occlusion, eliminating the need for intermaxillary fixation, risk of puncture injury from wires, correction of occlusion and reduction of the fracture during surgery by its multidirectional movement. Nevertheless, correction of the occlusion without good reduction of the fracture can occur due to the guide of reduction being the occlusion and lack of step deformity at the inferior border of the mandible (closed reduction).

However, this technique is not free of complications; extraoral distractors can lead to scars on the skin at the place where the pin is fixed. During the process of application they can injure the marginal branch of the facial nerve inferior alveolar nerve, tooth germ, roots of the teeth, delayed ossification and pain in the temporomandibular joints [11].

In our study, one patient showed mild malocclusion and this most probably due to difficulty of obtaining good occlusion without intermaxillary fixation. And mild trismus in another patient from soft tissue trauma during pin insertion was observed. Therefore, caution must be taken during insertion of the pins. The use of the transbuccal trocar as a guide overlaying the safe zones and the full knowledge of the anatomy decreased the incidence of this complication.

Eight patients didn't accept this technique due to the shape of the distractor and its effect on the lifestyle of the patient and restriction on the daily movement and this is one of the limitations of the use of the external fixator. Five patients were treated with closed reduction with intermaxillary fixation and three patients were treated with ORIF.

It has been proposed that effective insertion techniques and subsequent nursing care of pin sites will reduce the frequency of pin site infections, loosening of fixation and osteomyelitis [12-14]. Many different regimens for pin site care have been described. These include regular cleansing with solutions such as hydrogen peroxide [15], 0.9\% normal saline or cooled boiled water [16]. At the Kurgan Ilizarov Institute in Russia a very specific dressing regimen of $70 \%$ alcohol and $0.2 \%$ chlorhexidine, is prescribed immediately postoperatively. In this regime pins are never left uncovered and care is carried out by hospital personnel on a weekly basis [17]. However, there is no consensus on the optimal frequency of pin site care, which ranges from daily [18] to weekly [19] or even fortnightly [17]. In the present study, we used regular daily cleansing with $70 \%$ alcohol and $0.2 \%$ chlorhexidine solution at the pin sites and no signs of infection or pin loosening were observed.

\section{Conclusion}

The hallmark of external pin fixation is that it is a traumatic soft tissue management. The quality of the bony reduction is not guaranteed as the fragment ends are not often surgically exposed. External fixation remains a potentially effective quick, reliable and simple method to treat mandibular fractures in selected clinical situations. The external use of the distractor limited its application for fixation of the comminuted mandibular fracture.

Further studies are required for the evaluation of the efficacy of the multidirectional extraoral distractor as an external pin fixator on a large number of subjects.

\section{References}

1. Finn RA. Treatment of comminuted mandibular fractures by closed reduction. J Oral Maxillofac Surg. 1996;54(3):320-327.

2. Al-Assaf DA, Maki MH. Multiple and comminuted mandibular fractures: treatment outlines in adverse medical conditions in Iraq. J Craniofac Surg. 2007;18(3):606- 
612.

3. Fonseca JR, Walker RV, Bettis JN, Dexter BH. Oral and maxillofacial trauma, vol 1. 2nd ed. Philadelphia: WB. Saunders Company; 1997.

4. Kuriakose MA, Fardy M, Sirikumara M, Patton DW, Sugar AW. A comparative review of 266 mandibular fractures with internal fixation using rigid $(\mathrm{AO} /$ ASIF) plates or mini-plates. Br J Oral Maxillofac Surg. 1996;34(4):315-321.

5. Prein J. Bone as material. Manual of internal fixation in the cranio-facial skeleton. Berlin: Springer-Verlag; 1998.

6. Ellis E, 3rd, Muniz O, Anand K. Treatment considerations for comminuted mandibular fractures. J Oral Maxillofac Surg. 2003;61(8):861-870.

7. Scolozzi P, Richter M. Treatment of severe mandibular fractures using AO reconstruction plates. J Oral Maxillofac Surg. 2003;61(4):458-461.

8. Stacey DH, Doyle JF, Mount DL, Snyder MC, Gutowski KA. Management of mandible fractures. Plast Reconstr Surg. 2006;117(3):48e-60e.

9. Uglesic V, Virag M, Aljinovic N, Macan D. Evaluation of mandibular fracture treatment. J Craniomaxillofac Surg. 1993;21(6):251-257.

10. Cornelius CP, Augustin JB, Sailer LK. External pin fixation for stabilization of the mandible--comeback of a method: historical review and first experiences with the 'mandible external fixator'. Oral Maxillofac Surg. 2009;13(1):1-14.

11. Albert T, Marijo B. Distraction Osteogenesis. Acta Stomatol Croat. 2002;36(1).

12. Kroll JA. The use of the 'fixateur externe' in infected fractures. Arch Chir Neerl. 1973;25(2):137-149.

13. Sisk TD. External fixation. Historic review, advantages, disadvantages, complications, and indications. Clin Orthop Relat Res. 1983;(180):15-22.

14. Green SA, Ripley MJ. Chronic osteomyelitis in pin tracks. J Bone Joint Surg Am. 1984;66(7):1092-1098.

15. Jones-Walton P. Clinical standards in skeletal traction pin site care. Orthop Nurs. 1991;10(2):12-16.

16. Sims M, Saleh M. Protocols for the care of external fixator pin sites. Prof Nurse. 1996;11(4):261-264.

17. Grant AD, Atar D, Lehman WB. Pin care using the Ilizarov apparatus: recommended treatment plan in Kurgan, Russia. Bull Hosp Jt Dis. 1992;52(1):18-20.

18. Tolo VT. External skeletal fixation in children's fractures. J Pediatr Orthop. 1983;3(4):435-442.

19. Ahlborg HG, Josefsson PO. Pin-tract complications in external fixation of fractures of the distal radius. Acta Orthop Scand. 1999;70(2):116-118. 